\title{
Myotonic lid lag in hypokalaemic periodic paralysis
}

\author{
JEROME S. RESNICK AND W. KING ENGEL \\ From the Medical Neurology Branch, National Institute of Neurological Diseases \\ and Blindness, National Institutes of Health, U.S. Public Health Service, Department \\ of Health, Education, and Welfare, Bethesda, Maryland, U.S.A.
}

Intermittent failure of muscle excitation and contractility occurs in the periodic paralyses. Affected individuals present clinically with recurrent episodes of skeletal muscle weakness, often with a family history of such attacks. Alteration in serum potassium during attacks has led to the designation of three types of periodic paralysis, namely, hypokalaemic, normokalaemic, and hyperkalaemic. The hyperkalaemic form is referred to as adynamia episodica hereditaria by Gamstorp in her description of such patients (Gamstorp, 1956). The response to administered potassium differs in the various forms of periodic paralysis: in the hypokalaemic type, exogenous potassium reverses the acute attack and is also prophylactic (Aitken, Allott, Castleden, and Walker, 1937); conversely, patients with the normokalaemic and hyperkalaemic types are potassium intolerant, i.e., administration of even modest doses of potassium precipitates an attack or aggravates one that has begun spontaneously (Gamstorp, 1956; Poskanzer and Kerr, 1961 ; van Der Meulen, Gilbert, and Kane, 1961; McArdle, 1962; van't Hoff, 1962). The potential hazard of confusing the hyper- and hypokalaemic types has been previously stressed, since the correct treatment of one is detrimental and potentially lethal to the other (Gamstorp, 1963).

Strength is characteristically normal between attacks in all three types of periodic paralysis. One sign that may be evident between attacks is myotonia, a feature known to occur in hyperkalaemic periodic paralysis (Gamstorp, 1963). The myotonia, often mild and variable, is most readily elicited in eyelids (myotonic lid lag), periorbital muscles after strong eye closure, or by percussion of the tongue, extensor forearm or thenar muscles (McArdle, 1962). In recent years its diagnostic value and therapeutic implications have been emphasized. McArdle (1963) states that ' . . . attacks occurring during the day on resting after exercise is characteristic of hyperkalaemic periodic paralysis; if evidence of motonia can be elicited, the diagnosis can be made with confidence'. He has reported a family with hyperkalaemic periodic paralysis in which myotonic lid lag was a characteristic feature, between and during attacks, with myotonia less evident or absent in other muscles. Recently Layzer, Lovelace, and Rowland (1966), describing a family with the hyperkalaemic type, mention myotonic lid lag as a sign probably unique to that disease, van't Hoff has described nine members of a family with periodic paralysis in which myotonic lid lag was a prominent feature in each affected member between and during attacks. He considered their disease to be a variant closely related to hyperkalaemic periodic paralysis since intolerance to administered potassium was demonstrated, although spontaneous hyperkalaemia was not (van't Hoff, 1962).

The three patients described in this report exhibited clinical myotonia, showed hypokalaemic changes during episodes of weakness, and not only tolerated potassium but were benefited by its adadministration, therefore demonstrating that myotonia is not a reliable indicator of the type of periodic paralysis.

In this connexion, the normal range for serum potassium is taken as $3.5-5.5 \mathrm{mEq} . / \mathrm{l}$, and patients were considered to show hypokalaemic changes if the serum potassium level fell below $3.4 \mathrm{mEq} . / 1$. when weakness occurred (Fig. 1), and rose above $4.0 \mathrm{mEq}$./1. concomitant with clinical recovery. Furthermore, in order for these patients to be considered as having hypokalaemic periodic paralysis it was also required that they tolerate being loaded with potassium without developing any weakness. The serum potassium values mentioned below in the case descriptions are plotted in Figure 1.

\section{CASE DESCRIPTIONS}

CASE 1 (NIH 05-26 31) K.S., a 17-year-old white schoolboy, had his first attack at age 15 when he awakened one morning with weakness in all extremities. Within three hours he was quadriplegic and developed respiratory weakness requiring tracheotomy. Serum potassium was 


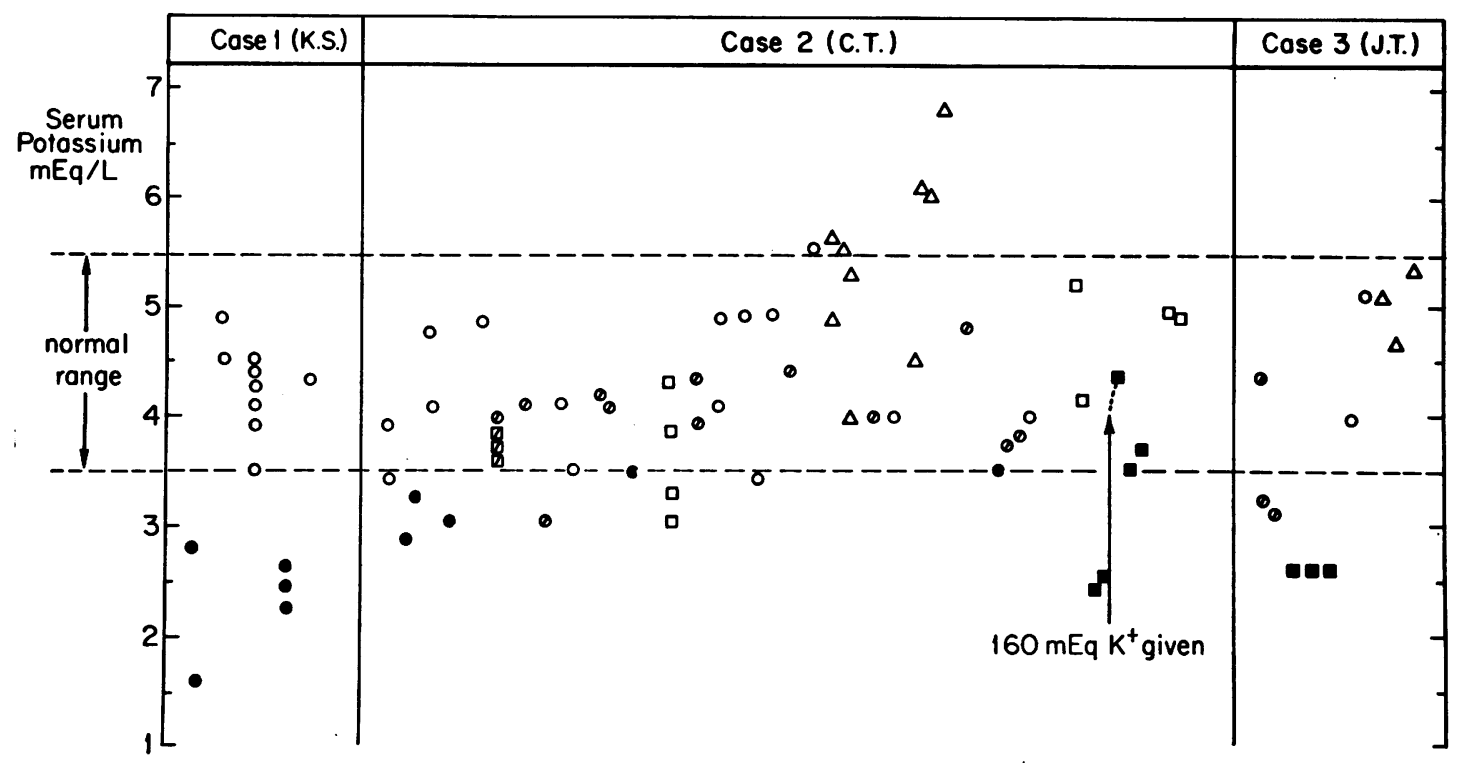

- No weakness.

a No weakness; glucose-insulin loading.

$\Delta$ No weakness; potassium loading.

- Mild weakness (no muscles lower than grade 4, MRC scale).

- Mild weakness; glucose-insulin loading.

$\Delta$ Mild weakness; potassium loading.

- Severe weakness (weakest muscles below grade 4, MRC scale).

- Severe weakness; glucose-insulin loading.

- Severe weakness; potassium loading.

FIG. 1. Serum potassium values as related to muscle power in the three cases described.

Explanation of third glucose-insulin loading of case 2: the white boxes indicate serum potassium levels of 5.3 and 4.3 during the first 30 minutes of the test when no weakness was present. At the end of 60 minutes paralysis occurred at which time potassium was 2.5 as indicated by black boxes. Treatment with $160 \mathrm{mEq} . \mathrm{K}^{+}$orally produced a temporary rise in serum potassium to 4.5 followed by another fall to 3.0 at which time weakness persisted. Five hours after the $\mathrm{K}^{+}$treatment was initiated, serum potassium reached 5.0 with concomitant recovery of full strength.

$1.6 \mathrm{mEq} . / 1$ and an E.G.C. showed hypokalaemic changes. He was treated with parenteral and oral $\mathrm{KC1}$, after which strength, serum potassium, and the E.C.G. returned to normal. During the next 18 months he experienced six episodes of mild morning weakness, for which he took oral potassium, with return of strength by noon. Two years after the first attack he had a second severe attack involving all extremities. He treated himself with oral potassium during the first two hours of this episode, but continued to get weaker and was therefore admitted to hospital that same morning, unable to walk, lift his arms, or support his head. During the first six hours in hospital strength improved slightly and serum potassium values were $2 \cdot 3,2 \cdot 4$, and $2.6 \mathrm{mEq}$./1. respectively at twohour intervals. Improvement continued, and the following morning strength was normal with a serum potassium value of $4.4 \mathrm{mEq} . / 1$.

Myotonic lid lag following sustained upward gaze was elicited on numerous occasions between attacks of weakness, and when present was striking. Usually the upper eyelids remained hung up for about 20 seconds after the patient looked rapidly down. Repeated up-and- down mvements of the eyes led to the disappearance of the myotonia for several minutes. Eyelid myotonia could be demonstrated about $75 \%$ of the time on initial testing. Occasionally percussion myotonia appeared in the thenar and extensor forearm muscles, but this was both less frequent and less prominent than in the eyelids. Myotonia was not evident during the second severe paralytic episode. It had not been looked for during his first attack. Between attacks Tensilon, $8 \mathrm{mg}$. intravenously, produced slurred speech and the sensation of 'thick tongue' for several minutes but did not increase the lid myotonia. Application of cold to the eyes did not increase the lid myotonia, nor did cooling of the face, arm, or tongue produce myotonia. Cold was not a precipitating factor of the paralysis. Thyroid function, aldosterone excretion, serum $\mathrm{Ca}, \mathrm{P}$, and $\mathrm{Mg}$ as well as other laboratory studies were unremarkable.

The patient had no family history of periodic paralysis. His only sibling, a 15-year-old girl, had two recent episodes when she was unable to release her grip from objects, requiring a companion to open her clenched fingers. She categorically denied weakness. Clinical 


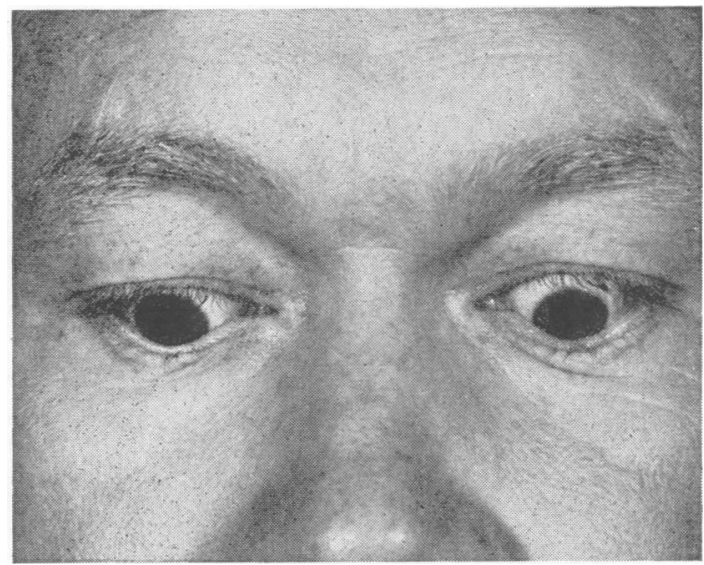

FIG. 2. Photograph taken after patient (case 2) looked down following prior upward gaze. Upper lids floated down 20 seconds after the picture was taken.

testing of the patient's mother and sister on two occasions revealed no evidence of myotonia.

CASE 2 (NIH 05-10-85) C.T., a 27-year-old white machine operator, had a history of episodic weakness in the trunk and extremities since the age of 13 . In severe attacks he could not roll over, sit, or stand. Onset was usually in the morning and was often preceded by hard physical exertion the previous day. The patient stated that sometimes he could 'walk off' an attack by continuing to exercise. Duration of the attacks varied from one hour to two days, with most lasting six to eight hours. These were not accompanied by dark urine. At times attacks occurred in clusters, almost daily for several weeks, and at other times they were weeks apart. Large meals and cold weather were not precipitating factors. Muscle stiffness during and between attacks was denied. The family history was positive as two of three brothers also had similar episodic weakness with onset in their teens. No ancestral history of the disorder was known, and offspring of the affected individuals were said to be normal.

On the initial admission to hospital no weakness was evident during the first three days, but myotonic lid lag was repeatedly elicited (Fig. 2) and shown to be worsened by local application of cold. Repetitive testing caused the lid myotonia to disappear. Tensilon did not alter the myotonia. Morning serum potassium levels were $3 \cdot 4$ and $3.9 \mathrm{mEq}$./1. on the second and third hospital days, when strength was normal. On the fourth hospital day the patient awakened with weakness in the neck and all extremities, more marked in distal extensor muscles which were unable to overcome gravity. The serum potassium level was $2.9 \mathrm{mEq}$./1. Myotonic lid lag was more pronounced, but myotonia could not be elicited anywhere else. No treatment was given and the following morning strength was somewhat better but still clearly below normal, with serum potassium $3.3 \mathrm{mEq} . / \mathrm{l}$. On the next morning, 48 hours after weakness began, strength was nearly normal and serum potassium levels were $4 \cdot 2$ and 4.6 mEq./l. That evening he exercised, and awakened the following morning again weak, with the triceps and hip flexors barely able to overcome gravity. The serum potassium level at that time was $3 \cdot 1 \mathrm{mEq}$./1. By midafternoon of the same day strength returned to normal spontaneously and the serum potassium level rose to $4.8 \mathrm{mEq}$./1. The lid myotonia did not consistently increase during episodes of weakness.

On another morning, two days after chlorothiazide (Diuril) 0.5 g. b.i.d., was begun, the serum potassium level was $3.1 \mathrm{mEq} . / \mathrm{l}$. and moderate weakness was present. Lid myotonia persisted. The patient was then given $75 \mathrm{mEq}$. of oral K $(5.6 \mathrm{~g}$. KC1) and within three hours strength improved. An additional $50 \mathrm{mEq}$. of K (3.8 g. $\mathrm{KC1}$ ) was given orally and the next morning strength was normal with a serum potassium level of $4 \cdot 2 \mathrm{mEq} . / 1$.

On several occasions this patient also had mild episodes of weakness during which serum potassium levels were not low, ranging from 4.0 to $4.8 \mathrm{mEq}$./., and during these the lid myotonia was evident, but no more so than before.

Oral potassium loading was administered on two occasions when the patient had no spontaneous weakness and when the serum potassium was in the normal range. No weakness occurred after $110 \mathrm{mEq}$. and $140 \mathrm{mEq}$. K (8.3 and $10.5 \mathrm{~g}$. KC1) on different days, although serum potassium levels rose to 5.7 and $6.8 \mathrm{mEq}$./1. within two hours following ingestion. The administration of $150 \mathrm{~g}$. glucose by mouth on one occasion, and intravenously on another, accompanied by 20 units regular insulin subcutaneously each time, did not produce weakness. However, profound weakness and a fall in serum potassium level from 5.3 to $2.5 \mathrm{mEq}$./1. occurred immediately after glucose $200 \mathrm{~g}$. and insulin 15 units were administered intravenously over a period of one hour. This provoked attack was treated with $160 \mathrm{mEq}$. oral $\mathrm{K}(12 \mathrm{~g}$. KC1), and several hours after treatment the serum potassium level rose to $5.0 \mathrm{mEq}$./l. with concomitant recovery of full strength. High sodium supplements $(260 \mathrm{mEq}$. $\mathrm{Na}$ in the form of $15 \mathrm{~g}$. NaCl by mouth daily for three days) had no apparent effect. Local cooling of the arm in ice water produced neither myotonia nor paralysis.

Thyroid function, serum $\mathrm{Ca}, \mathrm{P}$, and $\mathrm{Mg}$, as well as other laboratory studies, were unremarkable.

CASE 3 (NIH 05-20-72) J.T., a 27-year-old white male machine operator, complained of recurrent episodic weakness since the age of 20 , usually noted on awakening in the morning. All extremities could be involved, but the symptoms were usually more severe in the legs. Sometimes muscles felt stiff or cramped. The symptoms were not related to cold. Occasionally speech became slurred during severe attacks. Duration of symptoms was usually three but sometimes as long as seven days. The patient believed that attacks often occurred on the day following exercise and that continuing muscular activity afforded some relief. There was no history of dark urine. He had five siblings, none affected, and no ancestral history of neuromuscular disease.

Examination on the first hospital day revealed myotonic lid lag and bilateral Chvostek's sign, mild diffuse weakness, serum potassium levels of 4.4 and $3.2 \mathrm{mEq} . / 1$. and normal serum $\mathrm{Ca}, \mathrm{P}$ and $\mathrm{Mg}$ levels. The next morning 
the serum potassium level was $3.0 \mathrm{mEq} . / 1$. and strength was unchanged. Glucose, $50 \mathrm{~g}$. and insulin, 30 units intravenously, caused a fall in serum potassium to 2.6 $\mathrm{mEq}$./1. at which time he became dysarthric and limb weakness increased. The myotonic lid lag persisted and was, if altered, more pronounced during this induced attack. Local application of cold accentuated the lid myotonia but did not bring out this sign in any other muscles. On another day an oral glucose tolerance test was associated with transient increased weakness.

Oral potassium loading on four successive days with $37,110,110$, and $150 \mathrm{mEq}$. $\mathrm{K}(2 \cdot 8,8 \cdot 3$, and $11 \cdot 3 \mathrm{~g} . \mathrm{KC1})$ respectively produced no weakness. In fact, after the first loading, the previously persistent background of mild diffuse weakness lessened and the patient was stronger while on potassium. During the four days he received potassium, morning serum potassium levels were $4 \cdot 0,5 \cdot 1,5 \cdot 1$, and $4.6 \mathrm{mEq}$./l. respectively. The peak serum rise during the first few hours following potassium ingestion was not recorded. Chvostek's sign and lid myotonia bore no consistent relationship to the presence or absence of weakness.

\section{MUSCLE BIOPSY}

Biopsies of affected muscles were performed in all three patients during episodes of weakness. The tissue was divided into portions that were: (1) fixed (Zenker's, Boin's and formalin) for routine staining with haematoxylin-eosin, haematoxylin-Van Gieson, phosphotungstic acid haematoxylin, Gomori trichrome; (2) quick frozen for modified trichrome, periodic acid-Schiff and enzyme histochemistry, including myosin ATPase; phosphorylase; succinate, DPNH, TPNH, and lactate dehydrogenases; cytochrome oxidase; menadione-mediated a-glycerophosphate dehydrogenase, and esterase according to methods described elsewhere (Engel and Brooke, 1966).

CASE 1 Biopsy was performed on the right biceps within three hours of the onset of a spontaneous attack. The biopsied muscle was completely paralysed. No abnormalities were present. Six months earlier a biopsy had been taken between attacks from the left biceps with normal strength; the only abnormality consisted of 10 fibres with one to three small vacuoles each, in a $5 \mathrm{~mm}$. diameter fresh frozen specimen, which more accurately discloses subtle changes than does paraffin-embedded material.

CASE 2 Biopsy of the biceps was performed during a period when moderate weakness was present. Large single and multiple small 'mitochondrial aggregates' (Engel, 1964) appeared in 5 to $10 \%$ of the fibres, usually in subsarcolemmal locations. Apart from this, the muscle appeared unremarkable histologically but for a slight increase of internal nuclei. Specifically, vacuoles were not present.

CASE 3 Biopsy of the biceps was performed within two hours after glucose-insulin loading, at which time the muscle could move the joint through gravity but was unable to withstand other resistance. In a $5 \mathrm{~mm}$. diameter fresh frozen specimen, the only abnormalities were multiple small mitochondrial aggregates in 22 fibres and small vacuoles in 11 fibres.

\section{COMMENT}

The three patients described here clearly differ from other patients previously reported with the combination of periodic weakness and myotonic phenomena. Those described by McArdle (1962), Gamstorp (1956, 1963), van't Hoff (1962), van Der Meulen et al. (1961), and most recently, Layzer et al. (1966) were all thrown into attacks of weakness by oral potassium loading. By contrast, our patients did not show intolerance to potassium and in fact each one improved when potassium was given during periods of weakness.

The amounts of potassium given during loading trials in our cases 2 and 3 are comparable to the highest doses used by other authors to provoke attacks, and actually exceed the amounts that are usually required to precipitate weakness in hyperkalaemic periodic paralysis (McArdle, 1962; Gamstorp, 1963). Case 1 was not loaded with potassium while asymptomatic but has been treated successfully with over $100 \mathrm{mEq}$. K (7.5 g. KC1) in each of two acute spontaneous attacks when his serum potassium level was about $2 \mathrm{mEq}$./1. Also he takes supplemental daily oral $\mathrm{K}(3 \mathrm{~g}$. $\mathrm{KC1})$ prophylactically with apparent benefit.

The patients with myotonia and periodic paralysis described by other authors have shown the tendency to be weakest at those times when the serum potassium level was highest. In those patients the potassium level coincident with weakness is often at the high end of normal, rather than beyond absolute normal limits for each laboratory. In McArdle's family maximum strength was present only when serum potassium was $4.0 \mathrm{mEq}$./1. or lower (McArdle, 1962). In contrast, all three of our patients were weakest when the serum potassium level was low (below $3.5 \mathrm{mEq} . / 1$.) and strongest when $\mathrm{K}$ was above $4.5 \mathrm{mEq} . / 1$. This correlation of best strength with highest serum potassium levels occurred in our patients regardless of whether the higher serum potassium concentration developed spontaneously during recovery from an attack or was produced by administration of potassium. This is not to say that potassium levels fell every time that weakness developed in our patients. It should be noted that cases 2 and 3 were sometimes mildly weak when serum potassium was well within the normal range. However, when serum potassium did deviate outside it was not associated with serum potassium levels above $4.5 \mathrm{mEq} . / 1$. Cases 2 and 3 both developed 
acute, severe weakness and hypokalaemia with the glucose-insulin loading test.

Although the myotonia in eyelids was marked in our patients, none of them exhibited widespread myotonia, and electromyography of limb muscles did not show myotonic discharges. Case 1 at times had percussion myotonia in the extensor forearms and thenar mucles. None of the three patients showed percussion myotonia of the tongue. Their myotonic lid lag was impressive and on observation differed in no way from that present in hyperkalaemic periodic paralysis in which myotonia of the eyelid disappears after repeated rapid eye opening and closure, as occurred in our patients. Cooling usually enhances myotonia in hyperkalaemic periodic paralysis (and in other conditions where myotonia is present). However, worsening of myotonia by cold does not invariably happen, as noted by McArdle (1962) in one of his patients. In two of the three patients reported here, eyelid myotonia was exaggerated by cold.

Nevertheless, it might still be argued that the myotonic lid lag in our patients should not be considered true myotonia until electromyographic evidence of myotonia can be demonstrated. This consideration may be valid, but if adhered to, then some of the myotonia allegedly occurring in patients with hyperkalaemic periodic paralysis is also open to question, since in those reported it has frequently been confined to one or two muscles that do not lend themselves readily to routine electromyography, and has not appeared consistently. However, the concern of the present report is with a clinical sign which, regardless of whether or not it proves to be electrophysiologically substantiated myotonia, is indistinguishable at the bedside from the myotonia seen in hyperkalaemic periodic paralysis, and which heretofore has been considered an identifying feature when found in a patient with episodic weakness. The association of hyperkalaemic periodic paralysis and mytonia has received so much emphasis recently that, given a severely paralyzed patient in a situation where laboratory results on serum electrolytes are not immediately available, a physician might choose to assume that the disease is hyperkalaemic periodic paralysis and proceed with measures considered therapeutic, such as lowering serum $\mathrm{K}$. This could prove disastrous if, in fact, the patient had hypokalaemic periodic paralysis and had low serum potassium levels as in the cases reported here. Careful attention to the electrocardio- gram should also be stressed, since in some situations that may be more reliable than direct analysis of serum potassium.

Other noteworthy features of the patients described here are: as in hyperkalaemic periodic paralysis, two patients (cases 2 and 3) gave a clear history of weakness occurring during the period of rest after exercise and of sometimes being able to abort an attack by resuming physical activity. One patient had Chvostek's sign, a finding frequently present in hyperkalaemic periodic paralysis during attacks but not previously described in hypokalaemic periodic paralysis. The onset of the symptoms was during the second decade, a feature more typical of hyporather than hyper-kalaemic periodic paralysis. Unlike many patients with this condition, in none of our patients was cold a precipitating factor of weakness.

\section{SUMMARY}

Three patients are described having hypokalaemic periodic paralysis associated with prominent eyelid myotonia. These patients demonstrate that, contrary to other reports, the occurrence of myotonia in a patient with episodic weakness is not indicative of the hyperkalaemic form (adynamia episodica hereditaria) of periodic paralysis. The importance of not basing therapy for a patient with a periodic paralysis on the presence of myotonic lid lag is emphasized, because of possible disastrous consequences.

\section{REFERENCES}

Aitken, R. S., Allott, E. N., Castleden, L. I. M., and Walker, M. (1937). Observations on a case of familial periodic paralysis. Clin. Sci. 3, 47-57.

Engel, W. K. (1964) Mitochondrial aggregates in muscle disease. $J$. Histochem. Cytochem. 12, 46-48.

_- , and Brooke, M. H. (1966). Recent Advances in Neurological Diagnostic Methods. Thomas, Springfield, Ill.

Gamstorp, I. (1956). Adynamia episodica hereditaria. Acta paediat. (Uppsala), 45, suppl. 108, 1-24.

(1963). Adynamia episodica hereditaria and myotonia. Acta neurol. scand., 39, 41-58.

Layzer, R. B., Lovelace, R. E., and Rowland, L. P. (1966). Amer. Acad. Neurol., 18th annual meeting.

McArdle, B. (1962). Adynamia episodica hereditaria and its treatment. Brain, 85, 121-148.

_- (1963). Metabolic myopathies. Amer. J. Med., 35, 661-672.

Poskanzer, D. C., and Kerr, D. N. S. (1961). A third type of periodic paralysis, with normokalemia and favourable response to sodium chloride. Amer. J. Med., 31, 328-342.

van Der Meulen, J. P., Gilbert, G. J., and Kane, C. A. (1961). Familial hyperkalemic paralysis with myotonia. New Engl. J. Med., 264, 1-6.

van't Hoff, W. (1962). Familial myotonic periodic paralysis. Quart. J. Med., 31, 385-402. 